\title{
The phenomenon of degeneration of industrial Trichoderma reesei strains
}

\author{
R. Martzy ${ }^{1}$, T. M. Mello-de-Sousa' ${ }^{1}$ R. L. Mach², D. Yaver ${ }^{3}$ and A. R. Mach-Aigner ${ }^{1,2^{*}}$ (D)
}

\begin{abstract}
Background: Even if the loss of production capacity of a microorganism is said to be a serious problem in various biotechnology industries, reports in literature are rather rare. Strains of the genera Trichoderma reesei are used for large-scale production of cellulases, which are needed in food and feed, textile, paper industries and biofuel production.

Results: Here, we describe the phenomenon of spontaneous degeneration of T. reesei strains during large-scale cultivation. The phenotype of the degenerated population is characterized most importantly by a loss of any cellulase formation. Interestingly, promoter regions of relevant genes had a more compact chromatin in the (cel -) strains compared to productive strains. For a systematic investigation of the phenomenon a protocol for artificially induced and lab-scaled strain degeneration was developed. This workflow allows to determine the degeneration rate and thus, to compare the occurrence of a degenerated population in differently productive strains on the one hand, and to monitor the success of any strategies to prevent or decrease the degeneration on the other hand. While highly productive strains have higher degeneration rates compared to moderate producers, the degeneration can hardly be triggered in moderate producers. The observed (cel -) phenotype is not caused by a mutation in the gene encoding the essential transactivator Xyr1. The development of a non-producing population is also not triggered by any compounds released by either producing or non-producing cells.
\end{abstract}

Conclusions: The extent of the occurrence of a degenerated strain population relates to the production capacity of the strain and goes along with chromatin condensation in relevant promoter regions.

Keywords: Trichoderma reesei, Hypocrea jecorina, Filamentous fungi, Industry strains, Cellulases, Degeneration, Chromatin

\section{Background}

In nature, Trichoderma reesei is as a saprotrophic fungus an excellent producer of enzymes for break-down of plant biomass. In industry, these carbohydrate-active enzymes are used for a number of applications: for example, xylanases are used in the food industry as a baking agent and for clarification of juice and wine [1] or in the

\footnotetext{
*Correspondence: astrid.mach-aigner@tuwien.ac.at

${ }^{1}$ Christian Doppler Laboratory for Optimized Expression of Carbohydrate-Active Enzymes, Institute of Chemical, Environmental and Bioscience Engineering, TU Wien, Gumpendorfer Str. 1a, 1060 Vienna, Austria

Full list of author information is available at the end of the article
}

paper industry for de-inking [2]. Cellulases from T. reesei are applied in the textile industry, for example for fibre polishing [3] or in the paper industry for recycling processes [4]. For the production of ethanol from cellulosic raw material ("cellulose ethanol") cellulases obtained from $T$. reesei have a key role as well. In this process they are used to release D-glucose from the lignocellulosic biopolymers, which is subsequently used as carbon source in the sugar-to-ethanol fermentation (e.g., $[5,6]$ and citations therein).

Because of the broad application possibilities of $T$. reesei enzymes, and due to the outstanding secretion capacity of the fungus (around $100 \mathrm{~g} / \mathrm{L}$ [7]), T. reesei original author(s) and the source, provide a link to the Creative Commons licence, and indicate if changes were made. The images or other third party material in this article are included in the article's Creative Commons licence, unless indicated otherwise in a credit line to the material. If material is not included in the article's Creative Commons licence and your intended use is not permitted by statutory regulation or exceeds the permitted use, you will need to obtain permission directly from the copyright holder. To view a copy of this licence, visit http://creativecommons.org/licenses/by/4.0/. The Creative Commons Public Domain Dedication waiver (http://creativeco mmons.org/publicdomain/zero/1.0/) applies to the data made available in this article, unless otherwise stated in a credit line to the data. 
is used for industry-scale production of some carbohydrate-active enzymes. Genome-wide analysis of this fungus identified 34 genes coding for cellulolytic and xylanolytic enzymes (reviewed in [8]), of which the most prominent cellulases are the cellobiohydrolases CBHI and CBHII (EC 3.2.1.91) [9] and the most studied xylanases are the endo- $\beta-1,4-x y l a n a s e s \mathrm{XYNI}$ and XYNII (EC 3.2.1.8) [10]. The expression of the carbohydrate-active enzymes in $T$. reesei is regulated mainly at the transcriptional level in response to available carbon sources. Under glucose-limiting conditions the gene transcription is de-repressed; the full activation of cellulase production requires the presence of an inducing carbohydrate, e.g., cellulose or $\beta$-linked disaccharides such as cellobiose, sophorose, gentiobiose or lactose [11]. The full activation of xylanase expression is likewise dependent on the presence of xylan or xylanderived carbohydrates such as D-xylose or xylobiose [11].

The main and indispensable transactivator of both regulons (cellulase- and xylanase-encoding genes) is the Xylanase regulator 1 (Xyr1) $[12,13]$. Xyr 1 is a binuclear $\mathrm{Zn}$-cluster DNA-binding protein. It is generally expressed at a basal, rather low level; only by the cellulase expression-activating substance sophorose its own expression is also induced [14, 15]. Besides this, these regulons are either directly or indirectly subjected to carbon catabolite repression mainly mediated by the zinc finger protein Cre1. Cre1 does not only antagonize the transactivating function of $\mathrm{Xyr} 1$, it also represses xyr 1 expression itself. In this context, it is important to note that industry strains lack the full-length Cre1 protein $[16,17]$. This is a property that industrial stains inherited since most of them were obtained by several mutagenesis steps from the $T$. reesei strain Rut-C30 $[15,18]$. Rut-C30 is available for public research and was initially described as a hyper-cellulolytic strain [19, 20] and is itself a descendant of the T. reese $i$ wild-type QM6a.

During industry-scale cellulase production using hyper-productive $T$. reesei strains it occurs that the microorganism spontaneously loses productivity during the fed-batch fermentation process. Certainly, this poses a threat to the biotechnological production process because this degeneration phenomenon is not understood nor can it currently be prevented. In this study we detailed investigated the phenomenon of strain degeneration, i.e., the arising (cel -) phenotype in T. reesei for the first time, and shed some light on possible regulatory mechanisms. Besides, we developed a lab-scale protocol for artificially induced strain degeneration that enables a standardized investigation of the extent of degeneration, i.e., the degeneration rate. This protocol will also allow monitoring the success of any future measures aiming to reverse or prevent the degeneration of strains.

\section{Results}

The phenomenon of degeneration of industrial strains

$T$. reesei Iogen-M10 is a highly productive strain concerning cellulase formation. However, it can unpredictably happen during the cultivation process for cellulase production that it loses its productivity. An example for the occurrence of such degeneration phenomenon is displayed in Fig. 1. After approx. $72 \mathrm{~h}$ of cultivation, the specific productivity starts to decrease and at the same time a non-producing population rises. In course of the remaining cultivation time this (cel -) population quickly becomes the dominant population and usually, the more abundant the (cel - ) population becomes, the stronger the biomass formation increases (Fig. 1a, 144 and $168 \mathrm{~h}$ ). The isolation of non-producing colonies and the transfer to fresh full or inducing medium does not restore their cellulase production ability; therefore, the phenomenon needs to be considered as irreversible. Besides this, (cel -) cells possess a different morphology: hyphal cells are less bulbous and more elongated. According to Biolog investigations, they also have a reduced ability to metabolize cellulase-inducing carbohydrates such as lactose, cellobiose as well as several other carbon compounds, such as xylose, ribose, or arabinose. A third characteristic is that the occurrence of a (cel -) population is unpredictable, which makes any investigation difficult. However, observations over years suggested that better producing strains seem to degenerate more likely than moderately producing strains.

\section{The development of a (cel -) population might be driven by epigenetic mechanisms}

One obvious reason that could genetically cause the (cel -) phenotype would be a non-function mutation in the main transactivator Xyr1. However, a comparison of the genomic sequences of the xyr1 structure gene (including a 900-bp-long upstream region from ATG) between Iogen-M10 and its (cel -) counterpart strain did not reveal any differences. On the other hand, when degeneration occurs, besides productive cells and (cel -) cells, a third type of cell was isolated from mid- to latefermentation stages. These isolates form on acid-swollen cellulose (ASC) medium a smaller clearing zone compared to productive colonies, which form a large clearing zone, and a larger clearing zone compared to (cel -) colonies, which form no clearing zone at all (compare Fig. 1b). Serial passages of these (semi-cel -) cells to fresh ASC plates led to a slow loss of their ability to form any clearing zone, indicating the development to the full (cel -) phenotype. This gradual development from (semi-cel -) 

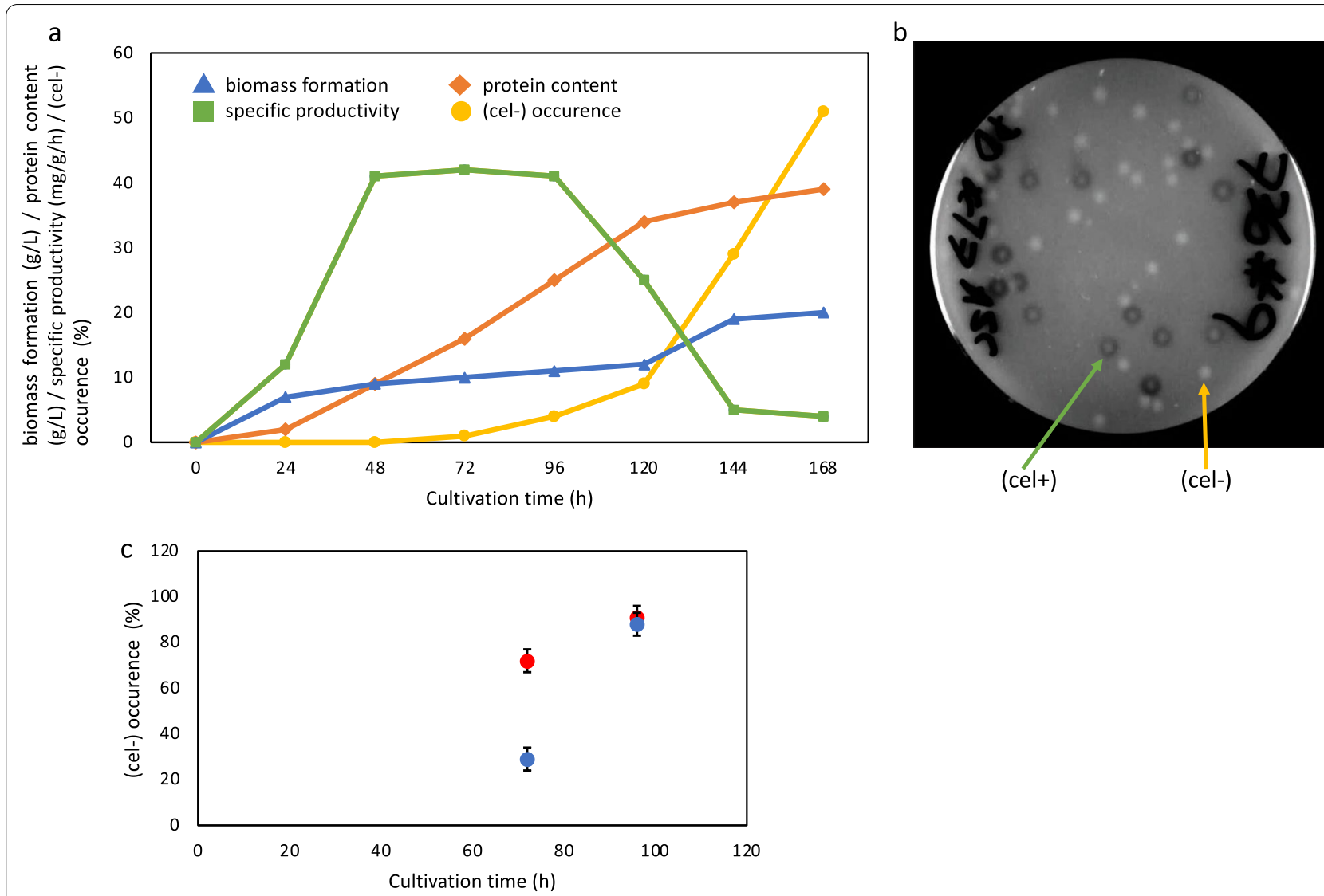

Fig. 1 Occurrence of a (cel -) population during cellulase production. T. reesei logen-M10 strain was cultivated in a 14-L bioreactor under cellulase producing conditions. a Samples were taken every $24 \mathrm{~h}$ and used for determination of biomass formation ( $\mathrm{g} / \mathrm{L}$; blue triangles), protein content ( $\mathrm{g} / \mathrm{L}$; red diamonds), specific productivity ( $\mathrm{mg} / \mathrm{g} / \mathrm{h}$; green squares) and cellulase activity [\% (cel -) population; yellow dots]. b For determination of cellulase activity the samples were spread on PDA plates for sporulation and then transferred to plates containing 1\% (w/v) ASC and incubated for $144 \mathrm{~h}$. The number of colonies without a halo, i.e., (cel -) colonies, and the number of colonies with a halo, i.e., (cel +) colonies were counted. c Determination of the development of a (cel -) population in samples after 72 and $96 \mathrm{~h}$ of cultivation in the presence (blue) and absence of (red) of Trichostatin A. Values are means of three replicates, error bars indicate standard deviations

to (cel -) suggests that this phenotype development could be the result of epigenetic mechanisms. For this reason, biomass samples, which were taken after $72 \mathrm{~h}$ and $96 \mathrm{~h}$ of the above-mentioned cultivation for cellulase production, were divided and spread on PDA plates and PDA plates containing additionally Trichostatin A. These samples were chosen because according to the cellulase activity tests the (cel -) population starts to develop within this time period (compare Fig. 1a). Trichostatin A is an inhibitor of histone deacetylation and is expected to prevent formation of heterochromatin. After growth and sporulation of the fungal cells on the PDA plates with and without Trichostatin A, spores were harvested and spread on ASC plates for determination of cellulase activity. Interestingly, in the case of the earlier taken sample $(72 \mathrm{~h})$ the $(\mathrm{cel}-)$ appearance could be prevented to a strong extent by the presence of Trichostatin A, while the later taken biomass sample ( $96 \mathrm{~h}$ ) exhibited the same high (cel -) population regardless whether it was treated with Trichostatin A (Fig. 1c). This is an indication that the development, however, not necessarily the initiation, of the (cel -) population might be driven by epigenetic mechanisms and could lead to higher heterochromatin formation in a (cel -) strain.

\section{The (cel -) phenotype is related to heterochromatin formation}

In order to test the above-mentioned speculation, we decided to use chromatin accessibility real-time PCR (CHART-PCR) assays to investigate the chromatin status of upstream regions of genes relevant for cellulase production. Due to its essential role for cellulase formation, we started with the analysis of three upstream regions of xyr1. One region was chosen as it might be 
a core region for transcription initiation (bearing a TATA-box-like element), one region was chosen due to a high number of regulatory cis elements, and a third region in between, which does not comprise any known element that is putatively relevant for cellulase formation, was included as well (Fig. 2a). Iogen-M10 and Iogen-M10 (cel -) were pre-grown and transferred to a condition inducing cellulase production (i.e., sophorose) and to a medium without any carbon source and were incubated for $3 \mathrm{~h}$. Chromatin compaction was found in the core region and the region bearing the regulatory cis elements in the (cel -) strain under both conditions, while the chromatin status of the middle region was similar in both strains under both conditions (Fig. 2b). To see whether the chromatin compaction that was observed in two regions in the (cel -) strain does affect transcript formation, we determined xyr 1 transcript levels by RT-qPCR. While no substantial change in $x y r 1$ transcript levels between Iogen-M10 and the (cel -) strain was found in case of the control condition (without any carbon source), under inducing conditions the $x y r 1$ transcript level was clearly lower in the (cel -) strain (Fig. 2c). The same experimental design was applied for promoter regions of the $c b h 1$ and $c b h 2$ genes. In both cases, two regions, a potential core region and a region further upstream which is strongly enriched in cis elements putatively relevant for cellulase expression, were investigated (Figs. 3a, 4a). For chb1, chromatin compaction was detected in the (cel -) strain in the core region under both conditions and in the upstream region under inducing conditions (Fig. 3b). A strong decrease in $c b h 1$ transcript was found for the (cel -) strain under both conditions compared to Iogen-M10 (Fig. 3c). For cbh2, chromatin compaction was detected in the (cel -) strain in both regions under both conditions (Fig. 4b). Transcript levels of $c b h 2$ were strongly reduced in the (cel -) strain compared to Iogen-M10 under both conditions (Fig. 4c). Summarizing, the (cel -) strain has a more compact chromatin in promoter regions of genes responsible for cellulase production, which is reflected in strongly reduced transcript levels of these genes. The
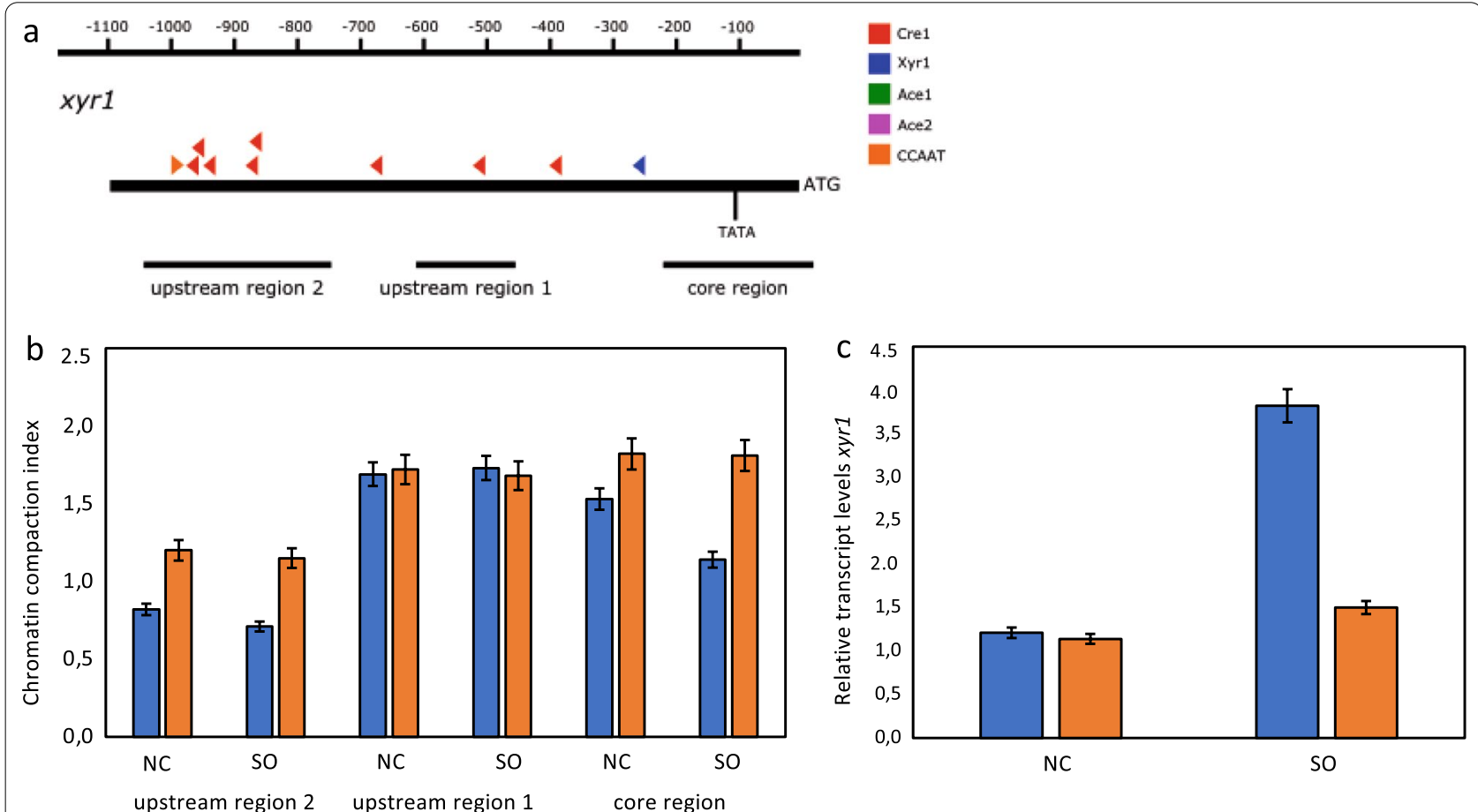

Fig. 2 Analysis of the chromatin status upstream of the xyr 1 gene and its transcript formation. a Schematic drawing of the upstream region of the xyrl gene. The black bars indicate the regions chosen for the CHART-PCR. Triangles indicate putative binding sites for transcription factors (colour-code provided). TATA indicates the position of a TATA-like box. Numbers on top indicate distance from ATG. T. reesei logen-M10 (blue bars) and logen-M10 (cel -) (orange bars) strains were pre-grown and transferred to sophorose (SO) or medium without carbon source (NC) and incubated for $3 \mathrm{~h}$. $\mathbf{b}$ CHART-PCR analysis of the three chosen regions. The chromatin compaction index (CCI) indicates the accessibility of DNA to DNase I digestion using normalization to sarl and act upstream regions. Higher CCI values indicate a more compact chromatin structure. $\mathbf{c}$ Transcript levels of xyr1. Transcript analysis was performed by RT-qPCR using sarl and act genes for normalization. Results are given as relative transcript ratios and refer to a reference sample (QM6a, NC). All values are the means of results from three independent experiments. Error bars indicate standard deviations 

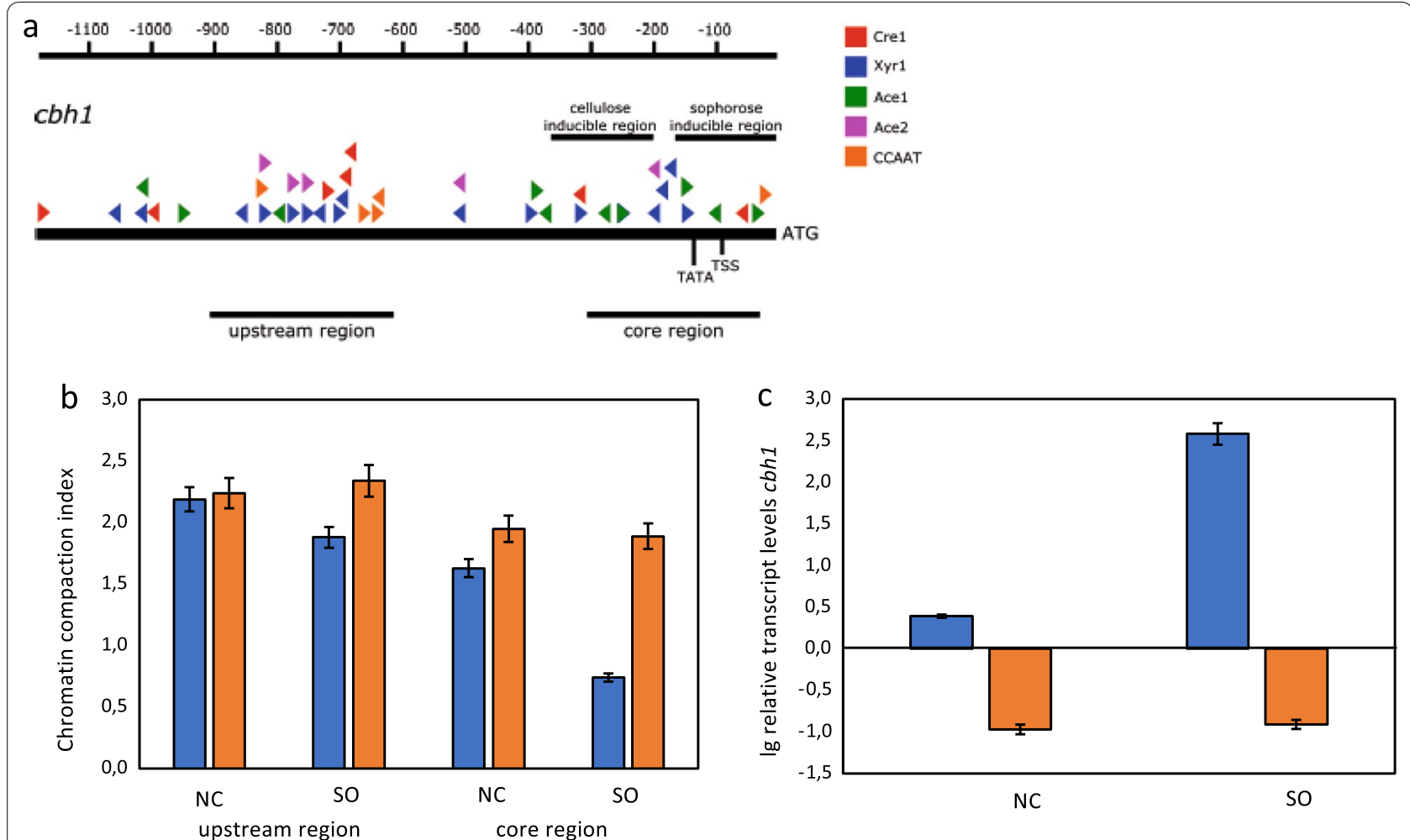

Fig. 3 Analysis of the chromatin status upstream of the cbhl gene and its transcript formation. a Schematic drawing of the upstream region of the cbh1 gene. The black bars indicate the regions chosen for the CHART-PCR. Triangles indicate putative binding sites for transcription factors (colour-code provided). TATA indicates the position of a TATA-like box. TSS indicates the position of the putative transcription start site. Numbers on top indicate distance from ATG. T. reesei logen-M10 (blue bars) and logen-M10 (cel -) (orange bars) strains were pre-grown and transferred to sophorose (SO) or medium without carbon source (NC) and incubated for $3 \mathrm{~h}$. $\mathbf{b}$ CHART-PCR analysis of the two chosen regions. The chromatin compaction index (CCl) indicates the accessibility of DNA to DNase I digestion using normalization to sar1 and act upstream regions. Higher CCI values indicate a more compact chromatin structure. c Transcript levels of cbh1. Transcript analysis was performed by RT-qPCR using sar 1 and act genes for normalization. Results are given as relative transcript ratios in logarithmic scale and refer to a reference sample (QM6a, NC). All values are the means of results from three independent experiments. Error bars indicate standard deviations

latter is in accordance with the observed loss of cellulase production in the (cel -) strain and suggests a relation of this phenotype to epigenetic mechanisms.

\section{A method for induced strain degeneration}

As mentioned above, observations indicated that different strains have a differently strong tendency to degenerate. Besides, it is obvious that any strategies for the preventing or reversing the degeneration phenomenon need a lab-scale format for a fast and standardized monitoring of the success of such strategies. For this purpose, a method was developed that allows the controlled trigger of strain degeneration and once the workflow is completed, it yields a degeneration rate, i.e., the percentage of (cel -) colonies within a whole population. Figure 5 displays the main workflow of the developed protocol for induced strain degeneration (ISD). Briefly, spores of the strain to be tested are generated on full medium. Harvested spores are split. One part is used to inoculate liquid minimal medium containing lactose as carbon source and perform an extended cultivation. Mycelium is harvested and again brought to sporulation on full medium. The obtained spores and the other part of the initial spores are used in a cellulase activity assay on plates, i.e., growth on minimal medium containing carboxymethyl cellulose as the only carbon source. This is followed by staining using Congo red for a clear visualization of a clearing zone (halo) around the colonies, which develops as the result of cellulolytic activity. The colonies with a halo are counted and related to the total number of colonies. For the development of the ISD protocol the production strain Iogen-M10 was used and samples were analysed every $24 \mathrm{~h}$ during the extended cultivation (Fig. 6a) and compared with the initial spores that were not submitted to the ISD protocol. As a control the strain, Iogen-M10 (cel -) was applied (Fig. 6a). This strain was isolated from a production process during which the strain degeneration phenomenon was observed. Figure 6 

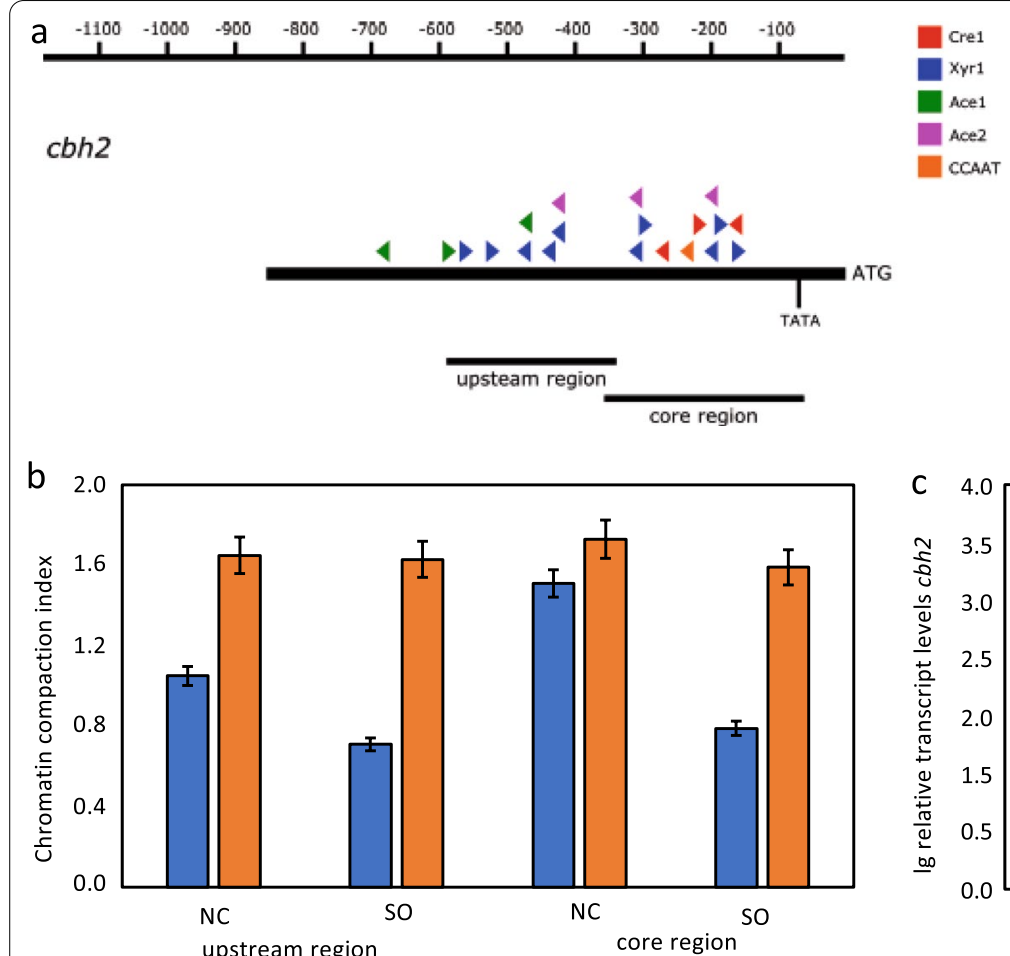

Fig. 4 Analysis of the chromatin status upstream of the cbh2 gene and its transcript formation. a Schematic drawing of the upstream region of the cbh2 gene. The black bars indicate the regions chosen for the CHART-PCR. Triangles indicate putative binding sites for transcription factors (colour-code provided). TATA indicates the position of a TATA-like box. Numbers on top indicate distance from ATG. T. reesei logen-M10 (blue bars) and logen-M10 (cel -) (orange bars) strains were pre-grown and transferred to sophorose (SO) or medium without carbon source (NC) and incubated for 3 h. $\mathbf{b}$ CHART-PCR analysis of the two chosen regions. The chromatin compaction index (CCI) indicates the accessibility of DNA to DNase I digestion using normalization to sarl and act upstream regions. Higher CCI values indicate a more compact chromatin structure. $\mathbf{c}$ Transcript levels of cbh2. Transcript analysis was performed by RT-qPCR using sarl and act genes for normalization. Results are given as relative transcript ratios in logarithmic scale and refer to a reference sample (QM6a, NC). All values are the means of results from three independent experiments. Error bars indicate standard deviations

displays the occurrence of the (cel -) population, which was already at $100 \%$ in the (cel - ) control strain (Fig. 6b) and developed over time in the producing strain until it also reached a uniform, non-producing population at the later stages of the ISD ( 96 h, 120 h) (Fig. 6c).

\section{The extent of degeneration relates to the productivity of a strain}

Since a protocol for standardized determination of the degeneration behaviour of a certain strain was now available, we decided to compare differently strongly evolved strains with regard to cellulase production. In this experiment we used the wild-type strain QM6a, strain Rut-C30, which was developed from QM6a by three rounds of mutagenesis and screening for increased protein secretion, Iogen-M4, which is a moderately producing industrial strain in the same strain lineage, and Iogen-M10, the before investigated, highly producing industrial strain, which also belongs to the same strain lineage (Fig. 7a). First, the total secreted protein was determined as an indicator for the productivity of the strains (Fig. 7b). The obtained results reflect the order of development of these strains for increased protein production. Second, the four strains were applied in the ISD protocol and degeneration rates were determined (Fig. 7c). While no degeneration was observed in case of the wild-type QM6a, a small (cel -) population developed in case of Rut-C30, an average degeneration rate of $10 \%$ was determined in case of Iogen-M4, and a rate close to $100 \%$ was found again in case of Iogen-M10 (Fig. 7c). Since no degeneration was observed for the wild-type strain at all, colonies were subjected to a second round of the ISD protocol to test whether the repeated exposure to cellulase-inducing conditions might more strongly trigger any degeneration. Thus, spores of all four strains after the first round of the ISD protocol were subjected to it a second time. Indeed, in case of the wild-type QM6a now low numbers of non-producing colonies were found, and in the case of Rut-C30 and Iogen-M4 a bigger (cel-) 


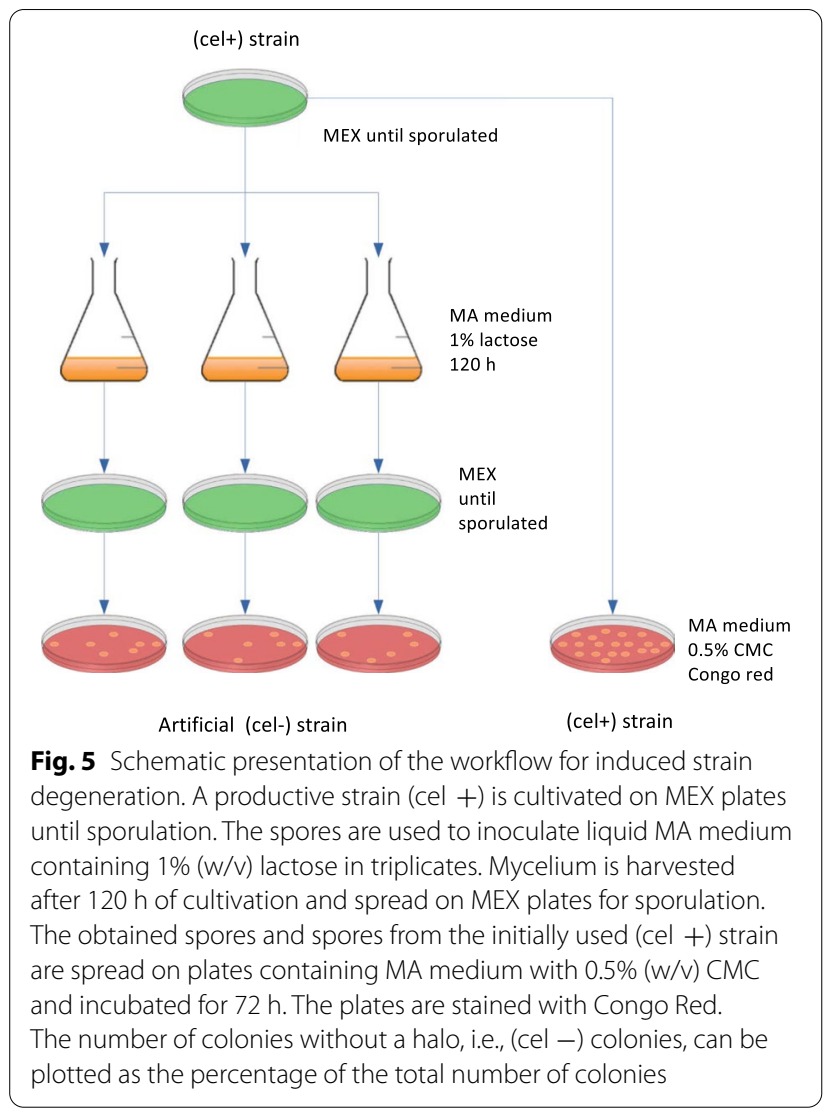

population developed. In the case of Iogen-M10 the (cel -) population was again close to $100 \%$ (Fig. 7c).

In relation to this finding, we also tested whether the presence of DTT changes the degeneration behaviour. For this purpose, we only used the hard-to-degenerate wild-type strain QM6a, again in two rounds of the ISD protocol with addition of DTT after $72 \mathrm{~h}$ of incubation. We observed the formation of a higher number of (cel -) colonies after the second round of the ISD in the presence of DTT (in average 11\%) compared to the absence of DTT in the earlier experiment (Fig. 7c). This finding together with the fact that higher productive strains have a stronger tendency to degenerate than moderately productive strains suggests a relation between degeneration phenomenon, productivity and ER stress (Fig. 7c).

\section{The development towards a (cel -) population is not driven by sensing any compounds}

We earlier in this manuscript reported that a (semicel -) population irreversibly develops towards a full (cel -) population. This prompted us to test whether either a highly productive or a degenerated strain might release any compound(s) that can be sensed by the productive cell and triggers mechanisms to turn into a nonproducing cell. To investigate this question, we used supernatants of cultivations of Iogen-M10 and its (cel -) counterpart to mix these conditioned media with fresh medium. For a detailed presentation of the experimental design, please refer to Fig. 8a. Strains that have different degeneration rates, namely QM6a, Rut-C30 and IogenM4, were submitted to the ISD protocol under standard conditions as control and using the mixed medium to test the possibility. Interestingly, for none of the strains a changed degeneration rate was found (Fig. 8b) indicating that it is not the presence of any compound that triggers the occurrence of the (cel -) population.

\section{Discussion}

Strain degradation is an often-observed phenomenon in eukaryotic microorganisms and spans from the loss of virulence of pathogenic microorganisms while studying them in the lab to the loss of productivity of industry strains used in biotechnology. Nonetheless, this phenomenon hampers basic research approaches as well as biotechnological processes. There are no reports about studies towards the understanding of the underlying mechanisms nor the prevention of this phenomenon.

One possible explanation for the occurrence of degeneration could be genomic imprinting. In this study, we found a relation between the degeneration rate of a strain and its productivity. Definitely, elevated stress caused for example by high levels of protein secretion, could be a crucial factor for the loss of productivity and the development of the (cel -) phenotype. Notably, it has been established in higher eukaryotes, as animals and plants, that epigenetic regulation and stress responses are closely interlinked (e.g., [21-23]). During the last two decades, quite some evidence was provided that cellulase expression in T. reesei is subject to epigenetic regulation through chromatin remodelling and/or DNA methylation. For example, the investigation of nucleosome positioning on the $c b h 2$ promoter of wild-type-like strains showed a dependence on the carbon source, i.e., cellulase-inducing versus repressing conditions [24]. This study provided indication that strain Rut-C30 loses a clear nucleosome positioning on the $c b h 2$ promoter independent of the carbon source used for cultivation. This led to the speculation that in Rut-C30 at least some parts of the genome (possibly including cellulase-encoding genes) are packed in a less condensed chromatin structure compared to other more wild-type-like strains. Later, Rassinger and co-workers reported an observation that reinforces this presumption: the $c b h 1$ promoter of Rut-C 30 cultivated on lactose has a stable open chromatin status while transcript levels are rising [25]. Our study suggests that the advantage of open chromatin in promoters of genes relevant for cellulase production, which was gained in this strain lineage, is lost in the (cel -) strains. 


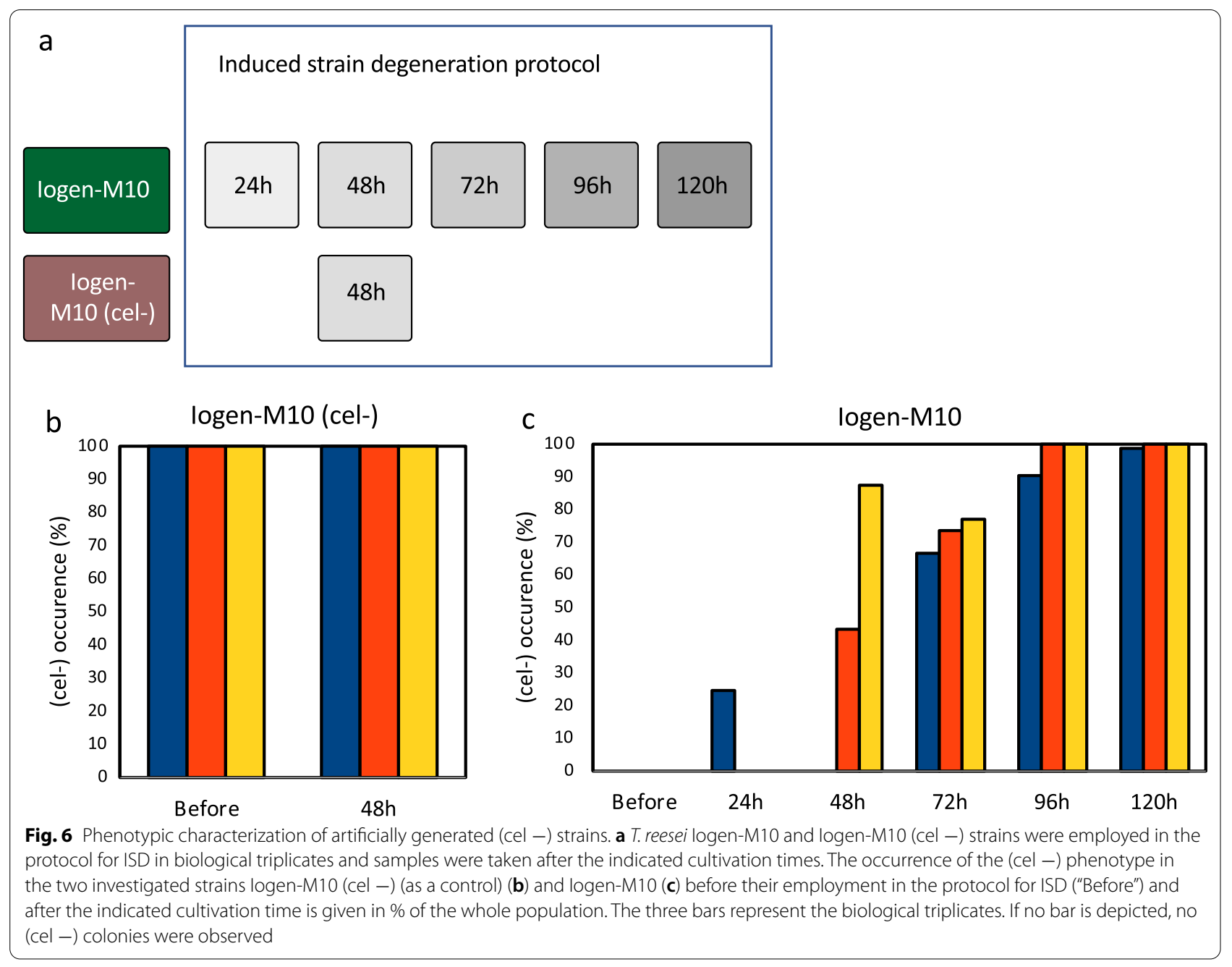

Another link between cellulase gene expression and chromatin structure was demonstrated in $T$. reesei strains possessing a deletion of the lae1 gene. Lae1 is the ortholog to laeA of Aspergillus spp., which recently was identified as a regulator of secondary metabolism [26] by acting as histone methyltransferase in chromatin remodelling (e.g., [27]). Deletion of Lae1 in T. reesei led to a loss of cellulase production, pointing to an influence of chromatin remodelling on the regulation of the cellulase expression [28].

Certainly, it remains the possibility that the (cel -) phenotype develops due to the occurrence and buildup of a genetic mutation. For example, early efforts for strain improvement by random mutagenesis did not only yield successful lineages as the one towards RutC30. Also strains without the ability to grow on cellulose or from cellulases even under inducing conditions were obtained (e.g., [29]). To identify possible genetic reasons for this phenotype, the whole genome of one of such strains, i.e., QM9136, was sequenced. For this strain, only a low number of mutations could be detected, however, one of them leading to a frameshift in $x y r 1$, which is highly likely the cause of the cellulase negative phenotype [30]. The possibility that a mutation in the xyr1 structural gene or upstream region might be responsible for the here described (cel -) phenotype was ruled out. Besides, it needs to be considered that the xyr1 frame shift in QM9136 led to an immediate loss of cellulase formation, while in case of the strain degeneration phenomenon, the (cel -) population gradually develops. Anyhow, only whole genome sequencing of a number of strains in the investigated Rut-C30 lineage and their respective (cel -) counterparts would allow to assess the impact of genetic mutations on the development of a degenerated population.

Altogether, only the understanding of the mechanism(s) causing the strain degeneration will give the possibility to prevent the undesired phenomenon. As discussed above several regulatory levels that 


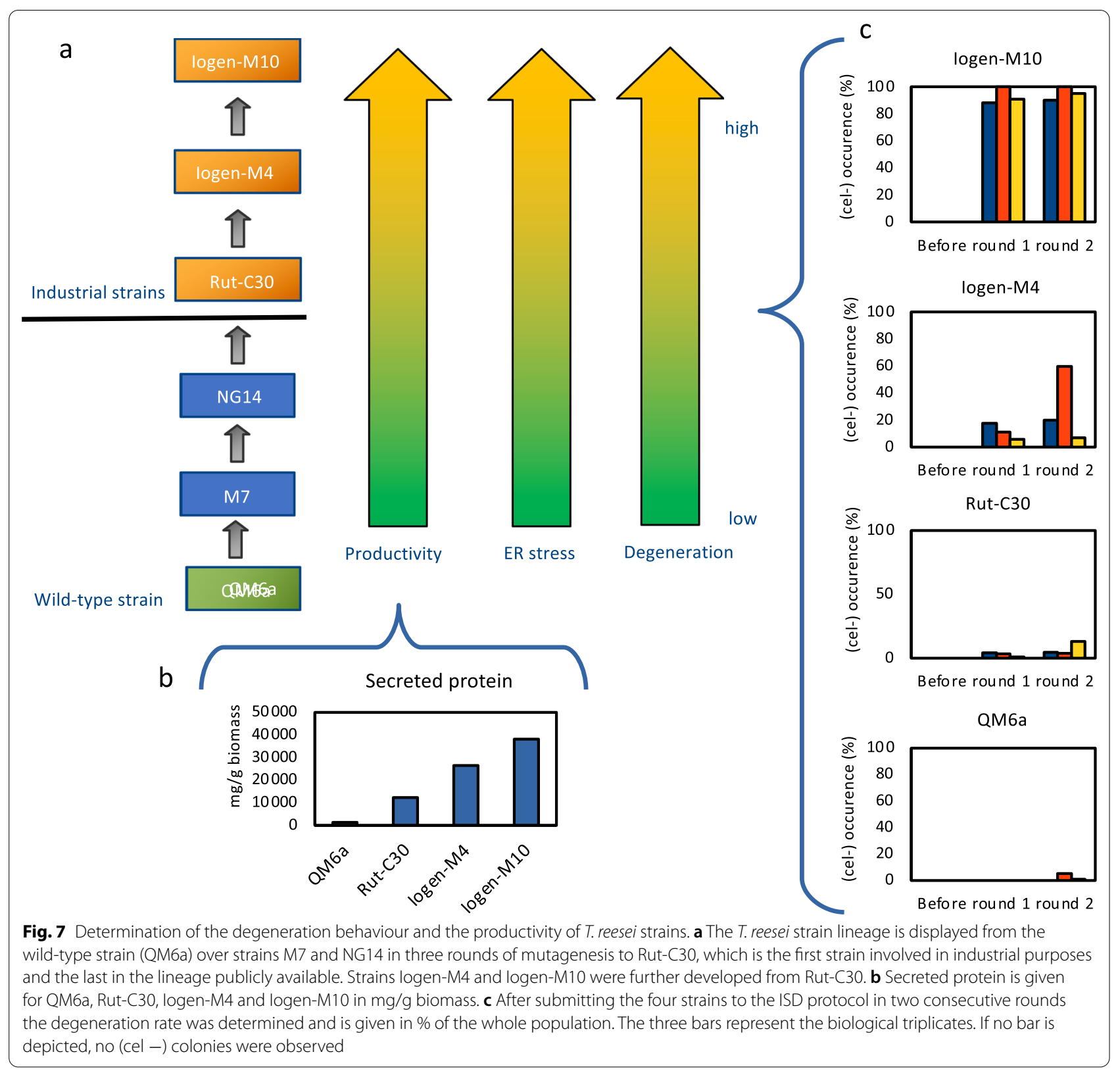

contribute to gene expression cannot be ruled out at the moment and warrant for further investigations.

\section{Conclusions}

The unpredictable development of a non-producing population of industrially used $T$. reesei strains during cultivation for cellulase production is a not understood phenomenon. The development of a protocol for ISD revealed that the rate of the degeneration correlates with the productivity of strains. Once turned into a (cel -) strain, the phenotype cannot be reversed, and (semi-cel -) strains gradually develop into the full (cel -) population suggesting the involvement of epigenetic mechanism. The chromatin of promoter regions of genes relevant for cellulase production become more condensed in (cel -) strains and transcript levels of the corresponding genes are strongly reduced. The impact of genetic mutations cannot be fully ruled out yet, however, mutations leading to a deficient Xyr1 can be excluded.

\section{Methods}

\section{Strains and cultivation conditions}

T. reesei Iogen-M4, Iogen-M4 (cel -) and Iogen-M10 and Iogen-M10 (cel -) strains (proprietary of Novozymes, 
a
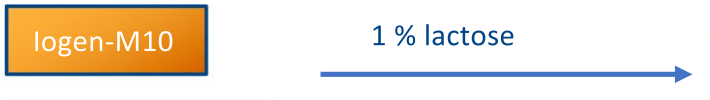

Conditioned medium, steril filtrated (CM)

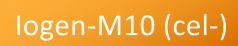

48 hours

\section{Conditioned medium, steril filtrated (CM)}

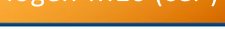

\section{CoMroga}

Rut-C30

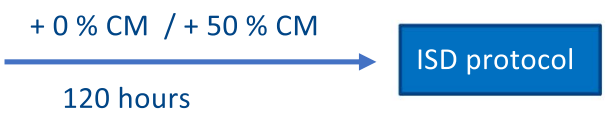

logen-M4

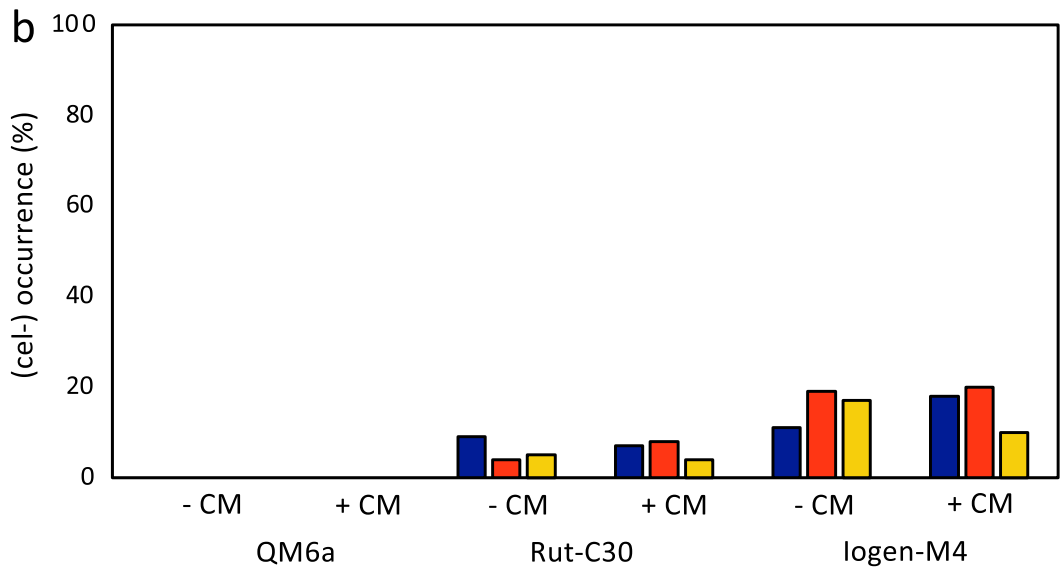

Fig. 8 Determination of the degeneration rate after growth in conditioned medium. a Schematic presentation of the experimental workflow. $T$. reesei logen-M10 and logen-M10 (cel -) strains were grown on 1\% lactose for $48 \mathrm{~h}$. Supernatants were sterile filtered and used to mix with fresh medium in the indicated ratio. T. reesei QM6a, Rut-C30 and logen-M4 strains were submitted to the ISD protocol under standard condition and using the mixed medium. $\mathbf{b}$ The degeneration rate was determined and is given in \% of the whole population. The three bars represent the biological triplicates. If no bar is depicted, no (cel -) colonies were observed

Bagsvaerd, Denmark) were maintained on potato dextrose agar (PDA) plates or on plates containing 3\% $(\mathrm{w} / \mathrm{v})$ malt extract, $0.1 \%(\mathrm{w} / \mathrm{v})$ peptone and $1.5 \%(\mathrm{w} / \mathrm{v})$ agar (MEX plates). For storage, the strains were kept on plates at $4{ }^{\circ} \mathrm{C}$ (short-term) or as spore suspensions in $25 \%$ glycerol at $-80^{\circ} \mathrm{C}$ (long-term).

Cultivation of $T$. reesei for enzyme production was performed in a 14-L pilot-scale bioreactor (Model MF114 New Brunswick, Eppendorf, Hamburg, Germany). For the inoculum, spores from a single PDA plate were transferred into $750 \mathrm{~mL}$ of liquid Berkley medium $(\mathrm{pH}$ 5.5) $\left[10.40 \mathrm{~g} / \mathrm{L}\left(\mathrm{NH}_{4}\right)_{2} \mathrm{SO}_{4}, 2.00 \mathrm{~g} / \mathrm{L} \mathrm{KH}_{2} \mathrm{PO}_{4}, 0.31 \mathrm{~g} / \mathrm{L}\right.$ $\mathrm{MgSO}_{4} \cdot 7 \mathrm{H}_{2} \mathrm{O}, 0.53 \mathrm{~g} / \mathrm{L} \mathrm{CaCl} \cdot 2 \mathrm{H}_{2} \mathrm{O}, 1 \mathrm{~mL} / \mathrm{L}$ trace element solution $\left(5 \mathrm{~g} / \mathrm{L} \mathrm{FeSO}_{4} \cdot 7 \mathrm{H}_{2} \mathrm{O}, 1.6 \mathrm{~g} / \mathrm{L} \mathrm{MnSO}_{4} \cdot \mathrm{H}_{2} \mathrm{O}\right.$, $\left.\left.1.4 \mathrm{~g} / \mathrm{L} \mathrm{ZnSO}_{4} \cdot 7 \mathrm{H}_{2} \mathrm{O}\right)\right]$ supplemented with $5.1 \mathrm{~g} / \mathrm{L}$ corn steep liquor powder and $10 \mathrm{~g} / \mathrm{L}$ glucose. The concentration of the inoculate for all cultivations or fermentations was $10^{6}$ spores $/ \mathrm{mL}$. Incubation was followed at $28{ }^{\circ} \mathrm{C}$ for 3 days at $100 \mathrm{rpm}$. The whole content was used as inoculum of 10-L Initial Pilot Medium (pH 5.5) [2.20 g/L $\left(\mathrm{NH}_{4}\right)_{2} \mathrm{SO}_{4}, 1.39 \mathrm{~g} / \mathrm{L} \mathrm{KH}_{2} \mathrm{PO}_{4}, 0.70 \mathrm{~g} / \mathrm{L} \mathrm{MgSO}_{4} \cdot 7 \mathrm{H}_{2} \mathrm{O}$, $0.185 \mathrm{~g} / \mathrm{L} \mathrm{CaCl}{ }_{2} \cdot 2 \mathrm{H}_{2} \mathrm{O}, 6.00 \mathrm{~g} / \mathrm{L}$ corn steep liquor powder, $13 \mathrm{~g} / \mathrm{L}$ glucose, $0.38 \mathrm{~mL} / \mathrm{L}$ trace element solution $\left(5 \mathrm{~g} / \mathrm{L} \mathrm{FeSO}_{4} \cdot 7 \mathrm{H}_{2} \mathrm{O}, 1.6 \mathrm{~g} / \mathrm{L} \mathrm{MnSO}_{4} \cdot \mathrm{H}_{2} \mathrm{O}, 1.4 \mathrm{~g} / \mathrm{L}\right.$ $\left.\left.\mathrm{ZnSO}_{4} \cdot 7 \mathrm{H}_{2} \mathrm{O}\right)\right]$. First, batch mode was used until the glucose was depleted, then, feeding with a solution containing cellulase-inducing carbohydrate (proprietary of Novozymes, Bagsvaerd, Denmark) was started at a carbon addition rate of $0.4 \mathrm{~g}$ carbon per $\mathrm{L}$ per $\mathrm{h}$. Cultivation was performed at $28^{\circ} \mathrm{C}$, an agitation rate of $500 \mathrm{rpm}$, and air sparging at 8 standard L per min for $168 \mathrm{~h}$. The $\mathrm{pH}$ was maintained at 4.0-4.5 during the batch mode and at $\mathrm{pH} 3.5$ during fed-batch using $10 \%(\mathrm{w} / \mathrm{v}) \mathrm{NaOH}$ solution. Samples of $100 \mathrm{~mL}$ culture broth were taken every $24 \mathrm{~h}$ and used for determination of biomass, protein content and cellulase activity. 
Cultivation of T. reesei for ISD was performed in $20 \mathrm{~mL}$ of Mandels-Andreotti (MA) medium containing 1\% $(\mathrm{w} / \mathrm{v})$ lactose and $0.1 \%(\mathrm{w} / \mathrm{v})$ peptone. After determining the spore count under a light microscope, $2 \times 10^{7}$ spores were used for inoculation and incubation of the cultures was followed at $30{ }^{\circ} \mathrm{C}$ and $160 \mathrm{rpm}$ for $120 \mathrm{~h}$. For the advanced degeneration, $20 \mathrm{mM}$ dithiothreitol (DTT) was added after $72 \mathrm{~h}$ and incubation was followed for $120 \mathrm{~h}$ in total. Cultures were harvested, regenerated on MEX plates and spores were eventually applied in a second round of ISD or directly used for determination of cellulase activity.

\section{Biomass determination}

For the gravimetric determination of the biomass content in samples from cultivation in a bioreactor, the constant weight of culture broth sample volumes of $5-10 \mathrm{~mL}$ was measured. Afterwards they were filtered using vacuum through glass fibre filters and oven-dried at $100{ }^{\circ} \mathrm{C}$ for $12 \mathrm{~h}$.

\section{Protein content determination}

The protein concentration in supernatants of the culture broth was determined by Bradford reagent followed by spectrophotometric measurement at $595 \mathrm{~nm}$. For quantification, a standard curve using a cellulase mixture of known composition was employed.

The specific productivity $\left(q_{p}\right)$ gives mg protein produced per $\mathrm{g}$ of biomass per hour of cultivation.

\section{Cellulase activity assay}

To determine whether or not the samples from cultivation in a bioreactor have cellulase activity, biomass samples were plated on PDA and incubated at $30{ }^{\circ} \mathrm{C}$ for 5 days until sporulation. In case of the investigation of the effects of Trichostatin A, $10 \mu \mathrm{M}$ were added to the plates. Spores were harvested and about 50-100 spores were spread on plates containing minimal medium and $1 \%(\mathrm{w} / \mathrm{v})$ acid-swollen cellulose (ASC) and $0.7 \%$ ox gall. These plates were incubated at $30^{\circ} \mathrm{C}$ for 6 days and afterwards at $50{ }^{\circ} \mathrm{C}$ for $20 \mathrm{~h}$. The formation of a clearing zone around a colony indicates cellulase secretion and activity. Cellulase producing and non-producing colonies were counted and the (cel -) occurrence was given as the percentage of non-producing colonies from the total number of colonies.

For determination of cellulase activity in samples from the ISD protocol, biomass samples were plated on MEX plates and incubated $30{ }^{\circ} \mathrm{C}$ for 5 days until sporulation. Spores were harvested and about 100-200 spores were spread on plates containing MA medium supplemented with $0.5 \%(\mathrm{w} / \mathrm{v})$ CMC (carboxymethylcellulose sodium salt, high viscosity), $0.1 \%(\mathrm{w} / \mathrm{v})$ peptone and $2.0 \%(\mathrm{w} / \mathrm{v})$ agar. These plates were incubated at $30{ }^{\circ} \mathrm{C}$ for 3 days and afterwards at $50{ }^{\circ} \mathrm{C}$ for $4 \mathrm{~h}$. Plates were stained with a $10-\mathrm{mL}$ aliquot of Congo red dye $(2.5 \mathrm{~g} / \mathrm{L})$ for $15 \mathrm{~min}$ at room temperature with gentle stirring, followed by washing the plates with $10 \mathrm{~mL}$ of $1 \mathrm{M} \mathrm{NaCl}$. The formation of a clearing zone around a colony indicates cellulase secretion and activity. Cellulase producing and non-producing colonies were counted and the (cel -) occurrence was given as the percentage of non-producing colonies from the total number of colonies.

\section{Biolog microarray technique}

The global carbon assimilation profiles were evaluated by using Biolog FF MicroPlate (Biolog, Inc., Hayward, CA, USA) following a previously described protocol [31], with minor modifications as follows: the inoculum was prepared from cultures on PDA plates incubated at $30{ }^{\circ} \mathrm{C}$. Mycelial growth was measured after 18, 24, 30, 36, 42, 48, 66,72 , and $90 \mathrm{~h}$ using biological triplicates.

\section{Sequencing of the $x y r 1$ locus}

The structural gene of $x y r 1$ and approximately $900 \mathrm{bp}$ upstream of the coding region were amplified as three overlapping fragments from 1200 to $1500 \mathrm{bp}$ using the Q $5^{\circledR}$ High-Fidelity DNA Polymerase (New England Biolabs, Ipswich, MA, USA). Primer sequences are provided in Table 1. The amplicons were purified from an agarose gel using the GeneJET Gel Extraction Kit (Thermo Fisher Scientific, Waltham, MA, USA) and cloned using the CloneJET PCR Cloning Kit (Thermo Fisher Scientific, Waltham, MA, USA). Fifty $\mu \mathrm{L}$ of TOP10 competent $E$. coli cells were transformed with the ligation mixes and plated on LB agar supplemented with ampicillin. Resulting single colonies were inoculated overnight in $5 \mathrm{~mL}$ of liquid LB medium supplemented with ampicillin and prepped using the GeneJET Plasmid Miniprep Kit (Thermo Fisher Scientific, Waltham, MA, USA). The plasmids were sent to Microsynth AG (Balgach, Switzerland) for an analysis by Sanger sequencing. All kits and reagents were used according to the manufacturers' instructions.

\section{CHART-PCR}

DNase I digestion of chromatin and DNA extraction were carried out as described by [32] with minor modifications. Mycelia were harvested by filtration, pressed dry with filter paper, frozen in liquid nitrogen, and ground to a fine powder. Portions $(100 \mathrm{mg})$ of the powder were suspended in 1-mL aliquots of nuclease digestion buffer $(250 \mathrm{mM}$ sucrose, $60 \mathrm{mM} \mathrm{KCl}, 15 \mathrm{mM} \mathrm{NaCl}$, $0.05 \mathrm{mM} \mathrm{CaCl}_{2}, 3 \mathrm{mM} \mathrm{MgCl}$, $0.5 \mathrm{mM}$ DTT, $15 \mathrm{mM}$ Tris- $\mathrm{HCl} \mathrm{pH} \mathrm{7.5),} \mathrm{and} 100-\mu \mathrm{L}$ samples of the digestion mixture were incubated with $10 \mathrm{U}$ of RQ1 RNase-free 
Table 1 Oligonucleotides used in this study

\begin{tabular}{|c|c|c|}
\hline Name & Sequence $\left(5^{\prime}-3^{\prime}\right)$ & Employment \\
\hline PCR1_fwd & TCCATCCCCATCCCGTTCTCCATCCATCCATG (32) & Sequencing of $x y r 1$ locus \\
\hline PCR1_rev & CTAAACAAGATCGATCAGTACATG (24) & \\
\hline PCR2_fwd & ATTCAACGGGTACTGCTGGG (20) & \\
\hline PCR2_rev & GTTCAAGTCGTGCTCATCCAC (21) & \\
\hline PCR3_fwd & CCACCTGCCAACCAGGAGG (19) & \\
\hline PCR3_rev & TAGAGGGCCAGACCGGTTC (19) & \\
\hline epiactinTr_f & СТTСССТССТТТССТСССССТССАС & CHART-PCR \\
\hline epiactinTr_r & GCGACAGGTGCACGTACCCTCCATT & \\
\hline epicbh1_1Tr_f & AAGGGAAACCACCGATAGCAGTGTC & \\
\hline epicbh1_1Tr_r & TTTCACTTCACCGGAACAAACAAGC & \\
\hline epicbh1_2Tr_f & GGATCGAACACACTGCTGCCTTTAC & \\
\hline epicbh1_2Tr_r & GGTTTCTGTGCCTCAAAAGATGGTG & \\
\hline epicbh2_1Tr_f & CGGATCTAGGGCAGACTGGGCATTG & \\
\hline epicbh2_1Tr_r & GTGTAGTGTTGCGCTGCACCCTGAG & \\
\hline epicbh2_2Tr_f & TGCAGCGCAACACTACACGCAACAT & \\
\hline epicbh2_2Tr_r & TGCGCCTCATACAGGGTCACAGTCC & \\
\hline episar1Tr_f & GTCAGGAAATGCCGCACAAGCAAGA & \\
\hline episar1Tr_r & TGTGTTTTACCGCCTTGGCCTTTGG & \\
\hline epixyr1_1Tr_f & CCTTTGGCCATCTACACAAGAGCAA & \\
\hline epixyr1_1Tr_r & CGCAATTTTTATTGCTGTTCGCTTC & \\
\hline epixyr1_2Tr_f & CCGACAGCAGCAGTAGTCAGGTTTT & \\
\hline epixyr1_2Tr_r & TAGGCAGAATAGCGACGGAGAGGAT & \\
\hline epixyr1_3Tr_f & GGCAGCCGTGTAGCTTGTCA & \\
\hline epixyr1_3Tr_r & GGAATCAAACCGTCGCCTCTT & \\
\hline act_fw & TGAGAGCGGTGGTATCCACG & qPCR \\
\hline act_rev & GGTACCACCAGACATGACAATGTTG & \\
\hline cbhif & GATGATGACTACGCCAACATGCTG & \\
\hline cbh1r & ACGGCACCGGGTGTGG & \\
\hline $\operatorname{cbh} 2 f$ & CTATGCCGGACAGTTTGTGGTG & \\
\hline $\mathrm{cbh} 2 \mathrm{r}$ & GTCAGGCTCAATAACCAGGAGG & \\
\hline sar1fw & TGGATCGTCAACTGGTTCTACGA & \\
\hline sar1rev & GCATGTGTAGCAACGTGGTCTTT & \\
\hline xyr1f & CCCATTCGGCGGAGGATCAG & \\
\hline xyr1r & CGAATTCTATACAATGGGCACATGGG & \\
\hline
\end{tabular}

DNase I (Promega, Madison, WI, USA) for $2.5 \mathrm{~min}$ at $37{ }^{\circ} \mathrm{C}$. The reaction was stopped by adding $100 \mu \mathrm{L}$ of $40 \mathrm{mM}$ EDTA, 2\% SDS, followed by two rounds of phenol-chloroform extraction and one round of chloroform extraction. Samples were then treated with $10 \mu \mathrm{g} /$ $\mathrm{mL}$ of RNase A for $15 \mathrm{~min}$ at $37^{\circ} \mathrm{C}$ and precipitated with ethanol. DNA pellets were suspended in $100 \mu \mathrm{l}$ of $5 \mathrm{mM}$ Tris- $\mathrm{HCl} \mathrm{pH} \mathrm{7.5.} \mathrm{A} \mathrm{control} \mathrm{without} \mathrm{DNase} \mathrm{I}$ was included to monitor endonuclease activity. qPCR analysis of the DNase I-treated samples was performed to measure the relative abundance of target regions. PCRs were performed in a Rotor-Gene Q system (Qiagen, Hilden, Germany). All reactions were performed in triplicate. The amplification mixture (final volume $20 \mu \mathrm{L}$ ) contained $10 \mu \mathrm{L}$ of $2 \times$ iQ SYBR Green Mix (Bio-Rad, Hercules, CA, USA), $200 \mathrm{nM}$ forward and reverse primers and $10 \mathrm{ng}$ of DNA. Primer sequences are provided in Table 1. Cycling conditions were as follows: 3 min initial denaturation at $95^{\circ} \mathrm{C}$, followed by 40 cycles of $15 \mathrm{~s}$ at $95{ }^{\circ} \mathrm{C}$ and $60 \mathrm{~s}$ at $60{ }^{\circ} \mathrm{C}$. The amount of intact input DNA of each sample was calculated by comparing the threshold values of the PCR amplification plots with a standard curve generated for each primer set using serial dilutions of genomic, undigested DNA. The chromatin compaction index (CCI) was defined as: 


$$
\mathrm{CCI}=\frac{\mathrm{Ds}}{(\mathrm{Dc} 1+\mathrm{Dc} 2) / 2},
$$

where Ds is the amount of intact DNA detected for a target region and $\mathrm{D} c 1$ and $\mathrm{D} c 2$ are the amounts of intact DNA detected for the promoter regions of sar1 and act, respectively, used as reference genes for normalization.

\section{Transcript analysis}

Fungal mycelia were homogenized in $1 \mathrm{~mL}$ of peqGOLDTriFast DNA/RNA/protein purification system reagent (PEQLAB VWR, Radnor, Pennsylvania, USA) using a FastPrep(R)-24 cell disrupter (MP Biomedicals, Santa Ana, CA, USA). RNA was isolated according to the manufacturer's instructions, and the concentration was measured using the NanoDrop 1000 (Thermo Fisher Scientific, Waltham, MA, USA). Reverse transcription of the isolated mRNA was carried out using the RevertAid ${ }^{\mathrm{TM}} \mathrm{H}$ Minus First Strand cDNA Synthesis Kit (Thermo Fisher Scientific, Waltham, MA, USA) according to the manufacturer's instructions.

Quantitative PCR (qPCR) was performed in a RotorGene Q system (Qiagen, Hilden, Germany). Reactions were performed in technical duplicates or triplicates. The amplification mixture (final volume of $15 \mu \mathrm{L}$ ) contained $7.5 \mu \mathrm{L}$ of $2 \times$ iQ SYBR Green Mix (BioRad, Hercules, California, USA), $100 \mathrm{nM}$ forward and reverse primer, and $2.5 \mu \mathrm{L}$ of cDNA (diluted 1:20). Primer sequences are provided in Table 1. Data normalization using sar1 and act as reference genes and calculations were performed as previously published [33].

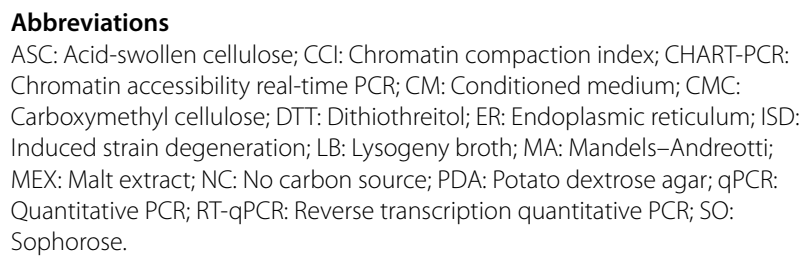

\section{Abbreviations}

ASC: Acid-swollen cellulose; CCl: Chromatin compaction index; CHART-PCR: Chromatin accessibility real-time PCR; CM: Conditioned medium; CMC: Carboxymethyl cellulose; DTT: Dithiothreitol; ER: Endoplasmic reticulum; ISD: Induced strain degeneration; LB: Lysogeny broth; MA: Mandels-Andreotti; MEX: Malt extract; NC: No carbon source; PDA: Potato dextrose agar; qPCR: Quantitative PCR; RT-qPCR: Reverse transcription quantitative PCR; SO: Sophorose.

\section{Acknowledgements}

Not applicable.

\section{Authors' contributions}

RM performed cultivation experiments and cellulase activity assays. TMMS developed the ISD protocol. DY and RLM contributed to design of experiments. ARMA designed the study and wrote the manuscript. All authors read and approved the final manuscript.

\section{Funding}

The financial support provided by the Christian Doppler Research Association, the Austrian Federal Ministry for Digital and Economic Affairs, the National Foundation for Research, Technology and Development, and Novozymes is gratefully acknowledged.

\section{Availability of data and materials}

All data generated or analysed during this study are included in this published article. Trichoderma reesei strains logen-M4 and logen-M10 are Novozymes' proprietary strains.

\section{Declarations}

Ethics approval and consent to participate Not applicable.

\section{Consent for publication}

Not applicable.

\section{Competing interests}

DY was employed at Novozymes, ARMA received funding from Novozymes.

\section{Author details}

${ }^{1}$ Christian Doppler Laboratory for Optimized Expression of Carbohydrate-Active Enzymes, Institute of Chemical, Environmental and Bioscience Engineering, TU Wien, Gumpendorfer Str. 1a, 1060 Vienna, Austria. ${ }^{2}$ Institute of Chemical, Environmental and Bioscience Engineering, TU Wien, Gumpendorfer Str. 1a, 1060 Vienna, Austria. ${ }^{3}$ Production Strain Technology, Novozymes Inc., Davis, CA, USA.

Received: 5 July 2021 Accepted: 19 September 2021

Published online: 01 October 2021

\section{References}

1. Galante YM, de Conti A, Monteverdi R. Application of Trichoderma enzymes in the food and feed industries. In: Harman Kubicek CPGE, editor. Trichoderma \& Gliocladium. London: Taylor \& Francis; 1998. p. 327-42.

2. Buchert J, Oksanen T, Pere J, Siika-aho M, Suurnäkki A, Viikari L. Applications of Trichoderma reesei enzymes in the pulp and paper industry. In: Harman GE, Kubicek CP, editors. Trichoderma \& Gliocladium. London: Taylor \& Francis Ltd.; 1998. p. 343-57.

3. Galante YM, de Conti A, Monteverdi R. Application of Trichoderma enzymes in the textile industry. In: Harman Kubicek CPGE, editor. Trichoderma \& Gliocladium. London: Taylor \& Francis; 1998. p. 311-25.

4. Oksanen T, Pere J, Paavilainen L, Buchert J, Viikari L. Treatment of recycled kraft pulps with Trichoderma reesei hemicellulases and cellulases. J Biotechnol. 2000;78:39-48.

5. Wilson DB. Cellulases and biofuels. Curr Opin Biotechnol. 2009;20:295-9.

6. Gusakov A. Alternatives to Trichoderma reesei in biofuel production. Trends Biotechnol. 2011;29:419-25.

7. Demain AL, Vaishnav P. Production of recombinant proteins by microbes and higher organisms. Biotechnol Adv. 2009. https://doi.org/10.1016/j. biotechadv.2009.01.008

8. Kubicek CP. Systems biological approaches towards understanding cellulase production by Trichoderma reesei. J Biotechnol. 2013;163:133-42.

9. Teeri SI, Knowles JT. The molecular cloning of the major cellulase gene from Trichoderma reesei. Biotechnology. 1983;1:696-9.

10. Törrönen A, Mach RL, Messner R, Gonzalez R, Kalkkinen N, Harkki A, et al. The two major xylanases from Trichoderma reesei: characterization of both enzymes and genes. Biotechnology. 1992;10:1461-5.

11. Margolles-Clark E, Ilmén M, Penttilä M. Expression patterns of ten hemicellulase genes of the filamentous fungus Trichoderma reesei on various carbon sources. J Biotechnol. 1997;57:167-79.

12. Stricker AR, Steiger MG, Mach RL. Xyr1 receives the lactose induction signal and regulates lactose metabolism in Hypocrea jecorina. FEBS Lett. 2007; 581:3915-20.

13. Stricker AR, Grosstessner-Hain K, Würleitner E, Mach RL. Xyr1 (xylanase regulator 1 ) regulates both the hydrolytic enzyme system and D-xylose metabolism in Hypocrea jecorina. Eukaryot Cell. 2006;5:2128-37.

14. Mach-Aigner AR, Pucher ME, Steiger MG, Bauer GE, Preis SJ, Mach RL. Transcriptional regulation of $x y r 1$, encoding the main regulator of the xylanolytic and cellulolytic enzyme system in Hypocrea jecorina. Appl Environ Microbiol. 2008;74:6554-62. 
15. Derntl C, Gudynaite-Savitch L, Calixte S, White T, Mach RL, Mach-Aigner AR. Mutation of the Xylanase regulator 1 causes a glucose blind hydrolase expressing phenotype in industrially used Trichoderma strains. Biotechnol Biofuels. 2013;6:62.

16. Strauss J, Mach RL, Zeilinger S, Hartler G, Stoffler G, Wolschek M, et al. Cre1, the carbon catabolite repressor protein from Trichoderma reesei. FEBS Lett. 1995;376:103-7.

17. IImén $M$, Thrane $C$, Penttilä $M$. The glucose repressor gene cre 1 of Trichoderma: isolation and expression of a full-length and a truncated mutant form. Mol Gen Genet. 1996;251:451-60.

18. Peterson R, Nevalainen H. Trichoderma reesei RUT-C30 - thirty years of strain improvement. Microbiology. 2012;158:58-68.

19. Montenecourt BS, Eveleigh DE. Preparation of mutants of Trichoderma reesei with enhanced cellulase production. Appl Environ Microbiol. 1977;34:777-82.

20. Montenecourt BS, Eveleigh DE. Production and characterization of high yielding cellulase mutants of Trichoderma reesei. TAPPI J. 1979;28:101-8.

21. Karpinets T, Greenwood D, Pogribny I, Samatova N. Bacterial stationarystate mutagenesis and mammalian tumorigenesis as stress-induced cellular adaptations and the role of epigenetics. Curr Genom. 2006. https:// doi.org/10.2174/138920206779315764.

22. Hauser M-T, Aufsatz W, Jonak C, Luschnig C. Transgenerational epigenetic inheritance in plants. Biochimica Biophysica Acta Gene Regul Mech. 2011. https://doi.org/10.1016/j.bbagrm.2011.03.007.

23. Chinnusamy $V$, Zhu J-K. Epigenetic regulation of stress responses in plants. Curr Opin Plant Biol. 2009. https://doi.org/10.1016/j.pbi.2008.12. 006.

24. Zeilinger S, Schmoll M, Pail M, Mach RL, Kubicek CP. Nucleosome transactions on the Hypocrea jecorina (Trichoderma reesei) cellulase promoter cbh2 associated with cellulase induction. Mol Genet Genomics. 2003;270:46-55.

25. Rassinger A, Gacek-Matthews A, Strauss J, Mach RL, Mach-Aigner AR. Truncation of the transcriptional repressor protein Cre1 in Trichoderma reesei Rut-C30 turns it into an activator. Fungal Biol Biotechnol. 2018. https://doi.org/10.1186/s40694-018-0059-0.

26. Bok JW, Keller NP. LaeA, a regulator of secondary metabolism in Aspergillus spp. Eukaryot Cell. 2004;3:527-35.

27. Reyes-Dominguez Y, Bok JW, Berger H, Shwab EK, Basheer A, Gallmetzer $A$, et al. Heterochromatic marks are associated with the repression of secondary metabolism clusters in Aspergillus nidulans. Mol Microbiol. 2010;76:1376-86.

28. Seiboth B, Karimi RA, Phatale PA, Linke R, Hartl L, Sauer DG, et al. The putative protein methyltransferase LAE1 controls cellulase gene expression in Trichoderma reesei. Mol Microbiol. 2012;84:1150-64.

29. Reese ET. History of the cellulase program at the US army Natick Development Center. Biotechnol Bioeng Symp. 1976;6:9-20.

30. Lichius A, Bidard F, Buchholz F, le Crom S, Martin J, Schackwitz W, et al. Genome sequencing of the Trichoderma reesei QM9136 mutant identifies a truncation of the transcriptional regulator XYR1 as the cause for its cellulase-negative phenotype. BMC Genomics. 2015. https://doi.org/10. 1186/s12864-015-1917-2.

31. Druzhinina IS, Schmoll M, Seiboth B, Kubicek CP. Global carbon utilization profiles of wild-type, mutant, and transformant strains of Hypocrea jecorina. Appl Environ Microbiol. 2006;72(3):2126-33.

32. Gonzalez R, Scazzocchio C. A rapid method for chromatin structure analysis in the filamentous fungus Aspergillus nidulans. Nucleic Acids Res. 1997;25:3955-6.

33. Steiger MG, Mach RL, Mach-Aigner AR. An accurate normalization strategy for RT-qPCR in Hypocrea jecorina (Trichoderma reesei). J Biotechnol. 2010;145:30-7.

\section{Publisher's Note}

Springer Nature remains neutral with regard to jurisdictional claims in published maps and institutional affiliations.
Ready to submit your research? Choose BMC and benefit from:

- fast, convenient online submission

- thorough peer review by experienced researchers in your field

- rapid publication on acceptance

- support for research data, including large and complex data types

- gold Open Access which fosters wider collaboration and increased citations

- maximum visibility for your research: over $100 \mathrm{M}$ website views per year

At BMC, research is always in progress.

Learn more biomedcentral.com/submissions 